\title{
Are the hotspots of radio galaxies the sites of in situ acceleration of relativistic particles?
}

\author{
Gopal-Krishna $^{1}$, P. Subramanian ${ }^{1,2}$, P. J. Wiita ${ }^{3,4}$, and P. A. Becker ${ }^{5}$ \\ 1 National Centre for Radio Astrophysics/TIFR, Pune University Campus, Post Bag No. 3, Ganeshkhind, \\ Pune 411007, India \\ e-mail: krishna@ncra.tifr.res.in \\ 2 Inter University Centre for Astronomy \& Astrophysics, Pune University Campus, Post Bag No. 4, Ganeshkhind, \\ Pune 411007, India \\ e-mail: psubrama@iucaa.ernet.in \\ 3 Department of Physics and Astronomy, Georgia State University, University Plaza, Atlanta, GA 30303-3083, \\ USA \\ e-mail: wiita@chara.gsu.edu \\ 4 Department of Astrophysical Sciences, Princeton University, Princeton NJ 08544-1001, USA \\ 5 Center for Earth Observing and Space Research, George Mason University, Fairfax, VA 22030, USA \\ e-mail: pbecker@gmu.edu
}

Received 6 February 2001 / Accepted 14 August 2001

\begin{abstract}
Using a large set of optically detected hotspots in powerful extragalactic double radio sources, we examine the basic question of whether the detection of optical synchrotron radiation requires in situ acceleration of relativistic electrons within the hotspots/lobes. For this, we take into account the jet's bulk relativistic motion, as well as its likely misalignment from the plane of the sky. Together, both these factors can drastically reduce the apparent range of the ultra-relativistic electrons ejected from the nucleus in the form of a jet. The conventionally adopted parameter space for the fundamental variables, namely, the hotspot magnetic field, radio source orientation angle relative to the line-of-sight and the bulk speed of the jet plasma, is considered. We find that the observed optical/near-IR synchrotron emission of the hotspots can be explained even if the radiating relativistic electrons were accelerated exclusively within the nuclear region, provided the energy losses incurred by the electrons during their transport down the jet are dominated by inverse Compton upscatterings of the cosmic microwave background photons. Under this circumstance, in situ acceleration of relativistic electrons inside the hotspots or lobes is not found to be mandated by their reported optical/near-infrared detections.
\end{abstract}

Key words. acceleration of particles - radiation mechanisms: non-thermal - galaxies: active - galaxies: jets radio continuum: galaxies - X-rays: galaxies

\section{Introduction}

A large fraction of the extra-nuclear radiation detected from radio galaxies is generally believed to arise from ultra-relativistic electrons with Lorentz factors $\gg 100$, undergoing synchrotron and/or inverse Compton losses. It is now well established that these electrons are initially accelerated in the vicinity of a compact supermassive object located at the active galactic nucleus, from where they are transported out to kiloparsec, or even megaparsec, distances in the forms of collimated jet-like features, giving rise to a pair of lobes of nonthermal emission on the opposite sides of the nucleus (e.g., Begelman et al. 1984; see,

Send offprint requests to: P. J. Wiita,

e-mail: wiita@chara.gsu.edu however, Valtonen \& Heinämäki 2000, for an alternative scenario). In powerful radio galaxies and quasars, the jets are seen to terminate in compact, bright regions, called hotspots, wherein much of the ordered kinetic power of the jet is presumed to be converted into random motion within the relativistic plasma and strengthened magnetic fields.

A long debated key issue is whether the ultrarelativistic electrons radiating within the kiloparsec-scale hotspots and lobes are largely directly supplied by the nucleus in the form of jets, or whether a significant amount of relativistic particle acceleration occurs in situ within the hotspots and lobes, for instance, near the shock fronts where the jet flow is thermalized (e.g., Scheuer 1989; Blandford \& Rees 1974). To effectively probe this 
question, many deep searches for optical/near-infrared (and even X-ray) radiation from hot spots have been carried out, motivated by the fact that the required electron Lorentz factors $\left(\gamma>10^{5}\right)$ correspond to a lifetime of $\lesssim 10^{3}$ yr against synchrotron losses, even in a magnetic field as weak as a few microgauss (e.g., Lelievre \& Wlerick 1975; Kronberg 1976; Saslaw et al. 1978; Simkin 1978; Meisenheimer \& Röser 1986; Crane et al. 1983; Röser $\&$ Meisenheimer 1987). While such early searches already led to positive detections of optical counterparts of a few radio hotspots, the subsequent detection of linear polarization firmly established the synchrotron nature of the optical hotspots (e.g., Meisenheimer \& Röser 1986; Röser \& Meisenheimer 1987; Hiltner et al. 1994; Thomson et al. 1995; Lähteenmäki \& Valtaoja 1999). X-ray detections of a few hot spots with ROSAT and CHANDRA have also been reported. However, in the absence of linear polarization measurements, attributing a synchrotron origin to the $\mathrm{X}$-ray emission is premature; in fact, an inverse-Compton origin or a synchrotron self-Compton explanation for the the X-ray emission is usually favored (e.g., Harris et al. 1994, 2000; Hardcastle et al. 2001; Wilson et al. 2000; see, however, Sect. 3 for the case of Pictor A radio lobe detected recently with $C H A N D R A$ ).

In a detailed analysis of the radio-to-optical synchrotron spectra of 8 hot spots associated with powerful, FR II (Fanaroff \& Riley 1974) radio galaxies, Meisenheimer et al. (1997) argued that the observed spectral cut-off in the frequency range $\nu_{\mathrm{c}}=3 \times 10^{11}-4 \times$ $10^{14} \mathrm{~Hz}$ can often be satisfactorily explained in terms of diffusive shock acceleration of relativistic electrons (up to the required $\gamma \sim 10^{5}$ ) at the strong terminal shock coincident with the hotspot. The theoretical framework for the in situ shock acceleration incorporating first-order Fermi acceleration has been developed in detail by many authors (e.g., Bell 1978; Biermann \& Strittmatter 1987; Drury 1983; Heavens \& Meisenheimer 1987; Kirk \& Schneider 1987). Some alternative proposals for production of ultrarelativistic electrons/positrons within the radio jets and lobes involve tapping, by various physical processes, the energy contained in a possible relativistic proton component of the beam flow (Dar \& Laor 1987; Mannheim et al. 1991; Mannheim 1993; Mastichiadis \& Kirk 1995; Subramanian et al. 1999), or a putative neutron component (Eichler \& Wiita 1978; Contopoulos \& Kazanas 1995), or simply, low-frequency electromagnetic waves (Rees 1971).

On the other hand, any model in which ultrarelativistic electrons are generated solely within the active nucleus must ensure their survival against the radiative losses suffered during their transit to the hotspot. Of the two principal energy loss mechanisms, namely, synchrotron and inverse-Compton emission, even the theoretically minimum loss scenario cannot evade the radiative losses arising from inverse-Compton scattering of the cosmic microwave background (CMB) photons. In this communication, we critically examine this question by taking into account the role of bulk relativistic motion of the jets, which is now widely believed to be maintained all the way out to multi-kpc-scales (e.g., Scheuer 1987; Laing 1988; Garrington et al. 1988; Bridle et al. 1994; Bridle 1996; Biretta et al. 1999) and which would lead to Dopplerenhanced CMB scattering losses. The visualization of the jets as the conduits of energy supply to the hotspots, in a manner largely free from synchrotron losses, has been discussed by several authors (e.g., Shklovskii 1984; Kundt 1986; Owen et al. 1989; Eilek et al. 1997), although its viability has been questioned by some others (e.g., Felton 1968; Meisenheimer 1996).

Nonetheless, since our aim here is to check if the need for in situ acceleration of electrons inside the hot spots is inescapable, we shall confine our attention to the energy losses that are absolutely unavoidable: the losses due to scattering of the CMB photons by the relativistic electrons flowing in the jet. (Note, however, that a minor loss of the jet power via synchrotron emission, particularly through interactions at the surface of the jet, is fully consistent with this picture; Sect. 3.) For visualizing the energy transport we shall confine ourselves to the conventional picture, whereby a jet consists of a quasi-continuous train of synchrotron plasmons in bulk relativistic motion, which radiate isotropically in their own frame of reference. It is also widely believed that the speeds with which the hotspots advance are non-relativistic (e.g., Scheuer 1995; Arshakian \& Longair 2000) and, consequently, any Doppler correction to their observed radiation is negligible. This avoids some of the uncertainties encountered while interpreting the jet emission.

We now focus attention on the thirteen radio galaxies and quasars for which the published high-resolution images have allowed determination of the radio-optical spectrum for at least one of the hotspots, thereby yielding a useful estimate of the spectral break frequency, $\nu_{\mathrm{c}}$ (Table 1). When optical and/or IR fluxes are found to be well registered with radio images of the hotspots, $\nu_{\mathrm{c}}$ can be fit to the radio through optical spectra using the method described in Meisenheimer et al. (1989). If the photometric measurements are made at multiple frequencies bracketing $\nu_{\mathrm{c}}$, its value can be determined to better than $50 \%$ (what we call Class I measurements), although if the measurements are further from $\nu_{\mathrm{c}}$, the error in its determination could be up to a factor of 2 to 3 (Class II); since our results for the maximum distances to which plasma can propagate only depends weakly on $\nu_{\mathrm{c}}$ (Eq. (6)), such uncertainties are acceptable. In a few cases, where multifrequency photometric data in the crucial optical/nearIR region are not available, we have taken, as a lower limit to $\nu_{\mathrm{c}}, 50 \%$ of the highest (optical/near-IR) frequency at which the hotspot has been detected; (these comprise the least certain, Class III, measurements). Still this assumption seems reasonable considering the steep decline that is seen to set in at $\nu_{\mathrm{c}}$ in the spectra of well detected hotspots (e.g., Meisenheimer et al. 1997). Our sample consists of 15 hotspots which are the prominent cases of optical synchrotron detections reported in the literature; however, a complete coverage of this literature is not claimed. 
In Col. (1) of Table 1 we give the source names, in Col. (2) whether it is a quasar $(\mathrm{Q})$ or a radio galaxy $(\mathrm{G})$, along with its redshift, $z$. Columns (3)-(5) give the equivalent magnetic field of the cosmic microwave background, measured in the frame of the jet for the three adopted values of the jet bulk Lorentz factor. Column (6) provides the angular separation of the hotspot from the nucleus. Columns (7) and (8) give the corresponding linear separation for different cosmological models. Column (9) notes whether polarization was detected in the optical hotspot, thereby cementing its synchrotron origin. Column (10) lists our best value for $\nu_{\mathrm{c}}$, and Col. (11) our estimate of the reliability of that value.

\section{The model}

A relativistic electron with Lorentz factor $\gamma$ on its way from the galactic nucleus to the hotspot loses energy to inverse Compton collisions with CMB photons of energy density, $u_{\mathrm{rad}, \mathrm{j}}$, and also suffers synchrotron losses in the transverse component of the jet field, $B_{\mathrm{j}}$. The energy-loss timescale of such an electron is (Longair 1981; Meisenheimer et al. 1989)

$\tau_{\mathrm{j}}=2.01 \times 10^{11} \gamma^{-1}\left[\frac{0.33 u_{\mathrm{rad}, \mathrm{j}}}{10^{6} \mathrm{eV} \mathrm{m}-3}+\left(\frac{B_{\mathrm{j}}}{\mathrm{nT}}\right)^{2}\right]^{-1} \mathrm{yr}$.

We stress that the quantities $u_{\mathrm{rad}, \mathrm{j}}, \tau_{\mathrm{j}}$, and $\gamma$ are measured in the jet frame. The energy density of the CMB photons in the "laboratory" frame (the frame in which the CMB radiation is isotropic) is (Scott et al. 2000):

$u_{\mathrm{rad}}=2.62 \times 10^{5}(1+z)^{4} \mathrm{eV} \mathrm{m}^{-3}$,

where $z$ denotes the cosmological redshift. In the frame of the jet, which is moving with bulk Lorentz factor $\Gamma_{j}$, the energy density of the impinging CMB photons is (e.g., Dermer \& Schlickeiser 1993, 1994; Begelman et al. 1994):

$u_{\text {rad }, \mathrm{j}} \simeq \Gamma_{\mathrm{j}}^{2} u_{\mathrm{rad}}$.

The equivalent magnetic field corresponding to $u_{\mathrm{rad}, \mathrm{j}}$ is $B_{\mathrm{CMB}}(\mathrm{nT})=0.325 \Gamma_{\mathrm{j}}(1+z)^{2}$ and is given in Table 1 for $\Gamma_{\mathrm{j}}=2,5$ and 10 .

Now, the synchrotron loss timescale, $\tau$, in the "laboratory" frame is related to that in the jet frame by the Doppler factor $\delta_{\mathrm{j}}$ :

$\tau=\delta_{\mathrm{j}}^{-1} \tau_{\mathrm{j}} \equiv \Gamma_{\mathrm{j}}\left(1-\beta_{\mathrm{j}} \cos \theta\right) \tau_{\mathrm{j}}$,

where $\beta_{\mathrm{j}}=\left(1-\Gamma_{\mathrm{j}}^{-2}\right)^{1 / 2}$ and $\theta$ is the angle between the line-of-sight and the direction connecting the core and the hotspot. In order to estimate the maximum range of the relativistic electrons, we follow Meisenheimer et al. (1989) in expressing the electron Lorentz factor, $\gamma$, in terms of "observables", using $\nu_{\mathrm{c}}=42\left(B_{\mathrm{HS}} / \mathrm{nT}\right) \gamma^{2}$, where $\nu_{\mathrm{c}}$ is the break frequency in the frame of the hotspot and $B_{\mathrm{HS}}$ is the magnetic field in the hotspot. (An averaging over pitch angles would yield a coefficient of 33 in place of 42 and would reduce the following results for $D_{\max }$ by
$11 \%$.) If we now set $B_{\mathrm{j}}=0$ (i.e., neglect synchrotron losses along the jet; cf. Sect. 1; see, however, Sect. 3), the maximum apparent (transverse) distance the jet's electrons can cover before dropping to less than $e^{-1}$ of their initial energy is given by:

$D_{\max }=\tau v_{\mathrm{app}}=\tau \frac{c \beta_{\mathrm{j}} \sin \theta}{\left(1-\beta_{\mathrm{j}} \cos \theta\right)}$.

We have used the usual expression for apparent transverse speed derived for explaining the superluminal expansion of VLBI cores (e.g., Rees 1967).

Substituting Eqs. (1)-(4) in Eq. (5) and solving for $\gamma$ in terms of $\nu_{\mathrm{c}}$ and $B_{\mathrm{HS}}$, we get

$D_{\max }=145 \frac{\beta_{\mathrm{j}} \sin \theta}{\Gamma_{\mathrm{j}}(1+z)^{4.5}}\left[\frac{\left(B_{\mathrm{HS}} / \mathrm{nT}\right)}{\left(\nu_{\mathrm{c}} / 10^{15} \mathrm{~Hz}\right)}\right]^{1 / 2} \mathrm{kpc}$.

Note that in Eq. (6), we have included in the denominator a factor of $(1+z)^{1 / 2}$ to correct for the cosmological redshift of $\nu_{\mathrm{c}}^{1 / 2}$. More precise calculations of Eq. (3) and the rate of energy loss, given in the Appendix, show that the results in Eqs. (4)-(6) should be multiplied by a factor of $\left(1+\beta_{\mathrm{j}}^{2} / 3\right) /\left(1+\beta_{\mathrm{j}}^{2}\right)$, which is always between $2 / 3$ and 1 .

To recapitulate, in the present analysis we have improved on the earlier treatments by making an allowance for both (i) the increased CMB energy density experienced by the jet plasma due to its bulk relativistic motion, and (ii) the offset of the radio axis from the plane of the sky, which is non-negligible in general. Consistent with the unified scheme for FR II radio sources (e.g., Barthel 1989; Gopal-Krishna et al. 1996), the approximate range of $\theta$ is $45^{\circ}-90^{\circ}$ for radio galaxies and $20^{\circ}-45^{\circ}$ for quasars, with blazars corresponding to the smallest angles.

In computing $D_{\text {obs }}$ in Table 1 , a Hubble constant $H_{0}=$ $75 \mathrm{~km} \mathrm{~s}^{-1} \mathrm{Mpc}^{-1}$ is assumed and two values of $q_{0}(=0.5$ and 0 ) are considered. We have used the usual relation (e.g., Lang 1999)

$D_{\mathrm{obs}}=\frac{c \Theta}{H_{0}} \frac{\left\{q_{0} z+\left(q_{0}-1\right)\left[\left(1+2 q_{0} z\right)^{1 / 2}-1\right]\right\}}{q_{0}^{2}(1+z)^{2}}$,

to relate $\Theta$, the observed angular separation between the central object and the hotspot (Col. 4 of Table 1), to $D_{\text {obs }}$ (Col. 5 of Table 1), the linear separation between the two, measured at the same redshift as is $D_{\max }$.

Using the data given in Table 1, we now compute for each hotspot the ratio $\eta \equiv D_{\text {obs }} / D_{\max }$, taking the usually adopted limiting values of the parameters $\theta, B_{\mathrm{HS}}$ and $\Gamma_{\mathrm{j}}$. The limiting values adopted for $B_{\mathrm{HS}}$ are $10 \mathrm{nT}$ and $100 \mathrm{nT}$, in that $35 \mathrm{nT}$ is the typical magnetic field strength in the hotspots (e.g., Meisenheimer et al. 1997). For $\Gamma_{j}$ we consider three values: $\Gamma_{\mathrm{j}}=2,5$ and 10 , which cover the range adopted in conventional models of the kpc-scale jets (e.g., Orr \& Browne 1982; Vermeulen \& Cohen 1994; Bicknell 1995; Wardle \& Aaron 1997). The computed values of $\eta$ for different combinations of the input parameters are plotted for each hotspot in Fig. 1. The relatively weak dependence of $D_{\max }$ on $\nu_{\mathrm{c}}$ means that even rather large errors in determining the break frequency do not have a major effect on our results. 
Table 1. Properties of the optical/near-infrared hotspots in the FRII radio sources*.

\begin{tabular}{|c|c|c|c|c|c|c|c|c|c|c|}
\hline $\begin{array}{c}\text { Source } \\
\text { Hotspot } \\
(1) \\
\end{array}$ & $\begin{array}{c}\text { Opt. ID } \\
z \text { (ref.) } \\
(2) \\
\end{array}$ & $\begin{array}{c}\Gamma_{\mathrm{j}}=2 \\
(3)\end{array}$ & $\begin{array}{c}B_{\mathrm{CMB}}(\mathrm{nT} \\
\Gamma_{\mathrm{j}}=5 \\
(4)\end{array}$ & $\begin{array}{c}\Gamma_{\mathrm{j}}=10 \\
(5)\end{array}$ & $\begin{array}{c}\Theta\left({ }^{\prime \prime}\right) \\
\text { (ref.) } \\
(6)\end{array}$ & $\begin{array}{c}D_{\mathrm{obs}}(\mathrm{kpc}) \\
q_{0}=0.5 \\
(7) \\
\end{array}$ & $\begin{array}{c}D_{\mathrm{obs}}(\mathrm{kpc}) \\
q_{0}=0 \\
(8) \\
\end{array}$ & $\begin{array}{c}\text { Opt. Pol. } \\
\text { (ref.) } \\
(9) \\
\end{array}$ & $\begin{array}{c}\nu_{\mathrm{c}}(\mathrm{Hz}) \\
(\text { ref.) } \\
(10) \\
\end{array}$ & $\begin{array}{c}\text { Class }^{\dagger} \\
(11) \\
\end{array}$ \\
\hline $\begin{array}{l}0003-003 \\
3 \mathrm{C} 2(\mathrm{~N})\end{array}$ & $\begin{array}{c}\mathrm{Q} \\
1.037(\mathrm{a})\end{array}$ & 2.7 & 6.7 & 11.0 & $\begin{array}{c}0.83 \\
(\mathrm{a}, \mathrm{b})\end{array}$ & 4.7 & 6.1 & - & $\begin{array}{l}\gtrsim 9 \times 10^{14} \\
\text { (a) }\end{array}$ & II \\
\hline $\begin{array}{c}0040+517 \\
3 \mathrm{C} 20(\mathrm{~W})\end{array}$ & $\begin{array}{c}\mathrm{G} \\
0.174(\mathrm{c})\end{array}$ & 0.9 & 2.2 & 3.7 & $\begin{array}{l}24.7 \\
\text { (c) }\end{array}$ & 62.9 & 65.7 & $\begin{array}{l}\text { detected } \\
\text { (d) }\end{array}$ & $\begin{array}{l}\sim 1.3 \times 10^{14} \\
\quad(\mathrm{c})\end{array}$ & I \\
\hline $\begin{array}{l}0106+130 \\
3 \mathrm{C} 33(\mathrm{~S})\end{array}$ & $\begin{array}{c}\mathrm{G} \\
0.0592(\mathrm{e})\end{array}$ & 0.7 & 1.8 & 3.0 & $\begin{array}{c}114 \\
(\mathrm{c}, \mathrm{f}, \mathrm{g})\end{array}$ & 118 & 120 & $\begin{array}{l}\text { detected } \\
(\mathrm{g})\end{array}$ & $\begin{array}{l}\sim 3.0 \times 10^{14} \\
\quad(\mathrm{c})\end{array}$ & I \\
\hline $\begin{array}{c}0415+379 \\
3 \mathrm{C} 111(\mathrm{E})\end{array}$ & $\begin{array}{c}\mathrm{G} \\
0.0485(\mathrm{c})\end{array}$ & 0.7 & 1.8 & 2.9 & $\begin{array}{c}122 \\
(\mathrm{c}, \mathrm{h}, \mathrm{i})\end{array}$ & 105 & 107 & $\begin{array}{l}\text { detected } \\
\text { (j) }\end{array}$ & $\begin{array}{l}\sim 1 \times 10^{14} \\
\quad \text { (c) }\end{array}$ & I \\
\hline $\begin{array}{c}0433+295 \\
3 \mathrm{C} 123(\mathrm{E})^{1}\end{array}$ & $\begin{array}{c}\mathrm{G} \\
0.2177(\mathrm{c})\end{array}$ & 1.0 & 2.4 & 3.9 & $\begin{array}{c}8.6 \\
(\mathrm{c}, \mathrm{h}, \mathrm{k}, \mathrm{l})\end{array}$ & 25.7 & 27.2 & - & $\begin{array}{l}\sim 3 \times 10^{11} \\
\quad \text { (c) }\end{array}$ & I \\
\hline $\begin{array}{c}0518-458 \\
\text { Pic A }(W)^{2}\end{array}$ & $\begin{array}{c}\mathrm{G} \\
0.0350(\mathrm{c})\end{array}$ & 0.7 & 1.7 & 2.8 & $\begin{array}{c}252 \\
(\mathrm{~m}, \mathrm{c}, \mathrm{h}, \mathrm{n})\end{array}$ & 161 & 163 & $\begin{array}{l}\text { detected } \\
\text { (o) }\end{array}$ & $\begin{array}{l}\sim 9 \times 10^{14} \\
\quad(\text { c) }\end{array}$ & I \\
\hline $\begin{array}{c}1056+432 \\
3 \mathrm{C} 247(\mathrm{~W})\end{array}$ & $\begin{array}{c}\mathrm{G} \\
0.749(\mathrm{p})\end{array}$ & 2.0 & 5.0 & 8.1 & $\begin{array}{c}\sim 9 \\
(\mathrm{q}, \mathrm{r})\end{array}$ & 49 & 59 & $\begin{array}{l}- \\
\text { (q) }\end{array}$ & $\begin{array}{l}\sim 1.3 \times 10^{14} \\
\quad(\mathrm{q})\end{array}$ & II \\
\hline $\begin{array}{c}1226+023 \\
3 \mathrm{C} 273(\mathrm{~S})^{3}\end{array}$ & $\begin{array}{c}\mathrm{Q} \\
0.158(\mathrm{c})\end{array}$ & 0.9 & 2.2 & 3.6 & $\begin{array}{l}21.3 \\
(\mathrm{~s})\end{array}$ & 50.4 & 52.5 & $\begin{array}{l}\text { detected } \\
(\mathrm{t})\end{array}$ & $\begin{array}{l}\sim 4 \times 10^{14} \\
(\mathrm{c}, \mathrm{h}, \mathrm{s})\end{array}$ & I \\
\hline $\begin{array}{c}1409+524 \\
3 \mathrm{C} 295(\mathrm{NW})^{4}\end{array}$ & $\begin{array}{c}\mathrm{G} \\
0.461(\mathrm{u})\end{array}$ & 1.4 & 3.5 & 5.7 & $\begin{array}{l}1.9 \\
(\mathrm{u})\end{array}$ & 8.7 & 9.8 & $\begin{array}{l}- \\
(\mathrm{u})\end{array}$ & $\begin{array}{l}\gtrsim 2 \times 10^{14} \\
\quad(\mathrm{u})\end{array}$ & $\mathrm{III}^{\ddagger}$ \\
\hline $\begin{array}{c}1441+522 \\
3 \mathrm{C} 303(\mathrm{~W})^{5}\end{array}$ & $\begin{array}{c}\mathrm{G} \\
0.141(\mathrm{c})\end{array}$ & 0.9 & 2.2 & 3.5 & $\begin{array}{c}16.6 \\
(\mathrm{c}, \mathrm{v}, \mathrm{q}, \mathrm{w})\end{array}$ & 36.0 & 37.3 & $\begin{array}{l}3 \sigma \\
(\mathrm{j})\end{array}$ & $\begin{array}{l}\sim 1.3 \times 10^{15} \\
\text { (c) }\end{array}$ & II \\
\hline $\begin{array}{c}1704+608 \\
3 \mathrm{C} 351(\mathrm{NE} 1)^{6}\end{array}$ & $\begin{array}{c}\mathrm{Q} \\
0.371(\mathrm{j})\end{array}$ & 1.2 & 3.1 & 5.0 & 25.8 & 106 & 117 & $2 \sigma$ & $\gtrsim 2 \times 10^{14}$ & $\mathrm{III}^{\ddagger}$ \\
\hline $3 \mathrm{C} 351\left(^{\mathrm{NE} 2)^{6}}\right.$ & $0.371(\mathrm{j})$ & & & & $\begin{array}{c}28 \\
(\mathrm{j}, \mathrm{w})\end{array}$ & 116 & 127 & $\begin{array}{l}2 \sigma \\
2 \sigma \\
(\mathrm{j})\end{array}$ & $\begin{array}{c}\gtrsim_{(j)} \\
{ }^{2} \times 10^{14}\end{array}$ & $\mathrm{III}^{\ddagger}$ \\
\hline $\begin{array}{c}1845+797 \\
\text { 3C } 390.3(\mathrm{~N})^{7}\end{array}$ & $\begin{array}{c}\mathrm{G} \\
0.0570(\mathrm{x})\end{array}$ & 0.7 & 1.8 & 3.0 & $\begin{array}{c}101 \\
(\mathrm{x}, \mathrm{y})\end{array}$ & 99.7 & 101 & $\begin{array}{l}1 \sigma \\
(\mathrm{j})\end{array}$ & $\begin{array}{c}\lesssim 3 \times 10^{14} \\
(\mathrm{y}, \mathrm{z}, \mathrm{j})\end{array}$ & II \\
\hline $\begin{array}{c}1957+406 \\
3 \mathrm{C} 405(\mathrm{~W})^{8}\end{array}$ & $\begin{array}{c}\mathrm{G} \\
0.0565(\mathrm{c})\end{array}$ & 0.7 & 1.8 & 3.0 & 67 & 66.1 & 67.0 & - & $\sim 10^{13}$ & II \\
\hline Cyg A $(E)^{8}$ & $0.0565(\mathrm{c})$ & & & & $\begin{array}{l}58 \\
(\alpha)\end{array}$ & 57.2 & 58.0 & - & $\begin{array}{c}\sim 10^{13} \\
(\mathrm{c}, \beta, \gamma)\end{array}$ & II \\
\hline
\end{tabular}

\section{Notes to Table 1:}

*: We have assumed a Hubble constant $H_{0}=75 \mathrm{~km} \mathrm{~s}^{-1} \mathrm{Mpc}^{-1}$.

$\dagger$ : Classes I, II and III represent decreasing confidence in the estimate of $\nu_{\mathrm{c}}$, depending upon the available spectral coverage for the hotspot. Class I denotes the cases where the measurements bracket $\nu_{\mathrm{c}}$ fairly tightly; class II where the multiple measurements lie to one side of $\nu_{\mathrm{c}}$, whereas for class III, the hotspot is detected only at a single optical/near-IR frequency (Sect. 1).

†: The adopted value of $\nu_{\mathrm{c}}$ is half the highest (optical/near-IR) frequency at which the hotspot has been detected (Sect. 1).

1: Detected only up to $230 \mathrm{GHz}$ (Meisenheimer et al. 1997).

2: Detected up to $\sim 5 \mathrm{keV}$ (Wilson et al. 2001).

3: Detected up to $\sim 5 \mathrm{keV}$ (Harris 2001; Röser et al. 2000).

4: Detected up to $\sim 5 \mathrm{keV}$ (Harris et al. 2000).

5: Resolved in I and K-band images (Lähteenmäki \& Valtaoja 1999; Meisenheimer et al. 1997; Keel 1988); there may be a knot in the jet (Meisenheimer et al. 1997).

6: Resolved in the $I$-band image; a double hotspot (Lähteenmäki \& Valtaoja 1999).

7: Resolved in I-band image ( $2^{\prime \prime}$, Lähteenmäki \& Valtaoja 1999; Saslaw et al. 1978). The spectrum is found to extend up to $\sim 2 \mathrm{keV}$ (Prieto \& Kotilainen 1997; Harris et al. 1998). However, a spectral break is apparent near $3 \times 10^{14} \mathrm{~Hz}(\mathrm{Harris}$ et al. 1998; Prieto \& Kotilainen 1997). This optical/near-IR hotspot appears to result from a collision of the jet with a dwarf galaxy which deflects the jet (Leahy \& Perley 1995; Harris et al. 1998).

8: Neither the eastern nor the western hot spot has a clear optical detection or a $K$-band counterpart (Meisenheimer et al. 1997). Both hotspots are detected in soft X-rays up to $5 \mathrm{keV}$ (Wilson et al. 2000; Harris et al. 1994).

References to Table 1: (a) Ridgway \& Stockton (1997); (b) Saikia et al. (1987); (c) Meisenheimer et al. (1997); (d) Hiltner et al. (1994); (e) Simkin (1979); (f) Dreher \& Simkin (1986); (g) Meisenheimer \& Röser (1986); (h) Meisenheimer et al. (1989); (i) Linfield \& Perley (1984); (j) Lähteenmäki \& Valtaoja (1999); (k) Okayasu et al. (1992); (l) Looney \& Hardcastle (2000); (m) Wilson et al. (2001); (n) Perley et al. (1997); (o) Thomson et al. (1995); (p) Polatidis et al. (1995); (q) Keel \& Martini (1995); (r) Jenkins et al. (1977); (s) Röser et al. (2000); (t) Röser \& Meisenheimer (1991); (u) Harris et al. (2000); (v) Kronberg et al. (1977); (w) Bridle et al. (1994); (x) Leahy \& Perley (1995); (y) Prieto \& Kotilainen (1997); (z) Harris et al. (1998); ( $\alpha$ ) Hargrave \& Ryle (1976); ( $\beta$ ) Wilson et al. (2000); $(\gamma)$ Harris et al. (1994). 

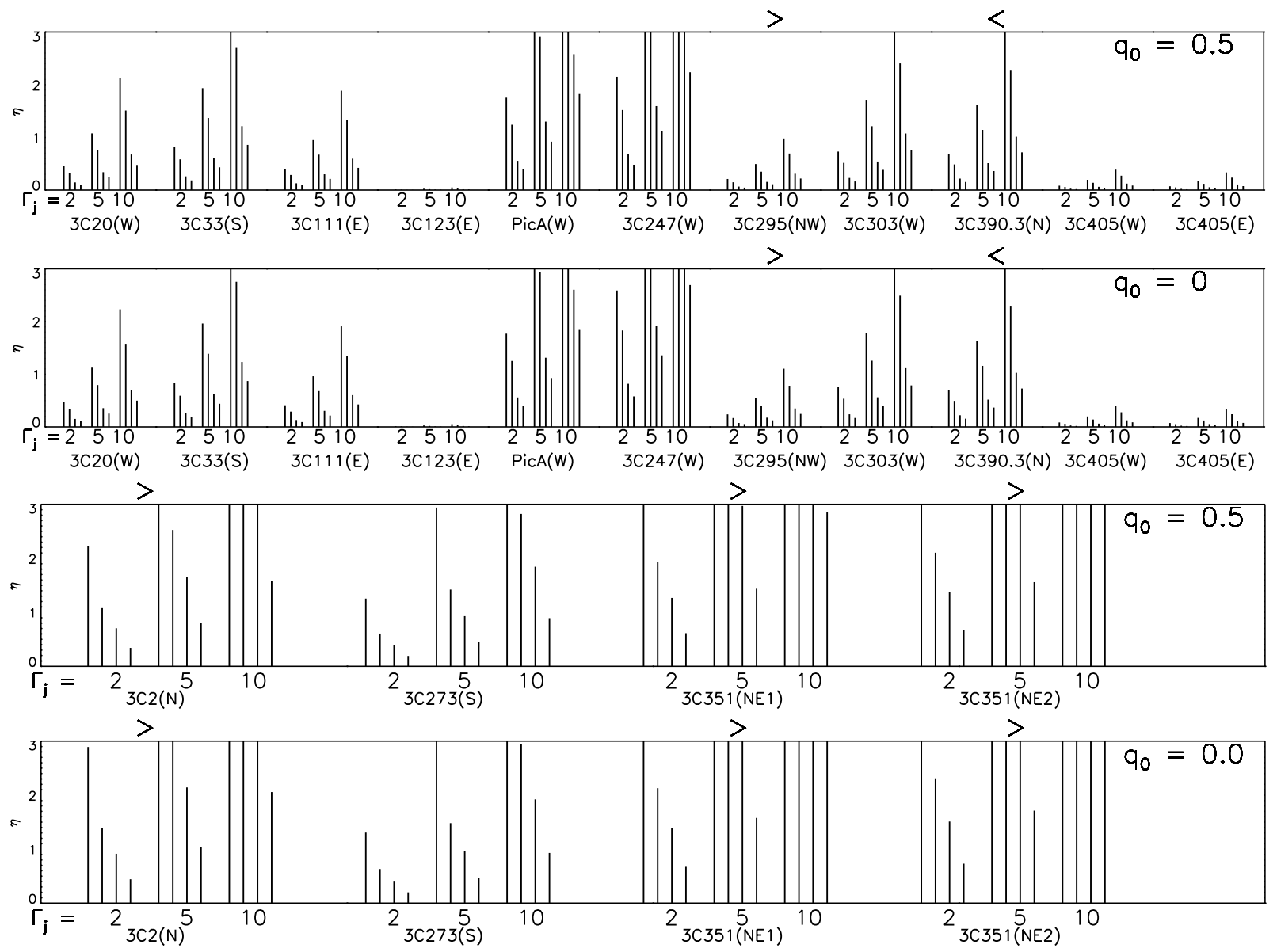

Fig. 1. The computed values of $\eta=D_{\text {obs }} / D_{\max }$ including the relativistic correction derived in the Appendix, for all 15 hotspots in our sample. The top two panels show the results for radio galaxies (for two values of $q_{0}$ ), while the results for the quasars are displayed in the bottom two panels. For each hotspot, there are three sets of four vertical lines each, representing the three values of the jet's bulk Lorentz factor $\Gamma_{\mathrm{j}}$. In the top two panels, each set of four vertical lines has the following combinations of $\theta$ and $B_{\mathrm{HS}}$, from left to right: $\left(\theta=45^{\circ}, B_{\mathrm{HS}}=10 \mathrm{nT}\right),\left(\theta=90^{\circ}, B_{\mathrm{HS}}=10 \mathrm{nT}\right),\left(\theta=45^{\circ}, B_{\mathrm{HS}}=100 \mathrm{nT}\right)$, $\left(\theta=90^{\circ}\right.$, $\left.B_{\mathrm{HS}}=100 \mathrm{nT}\right)$. The corresponding values for the bottom two panels are: $\left(\theta=20^{\circ}, B_{\mathrm{HS}}=10 \mathrm{nT}\right),\left(\theta=45^{\circ}, B_{\mathrm{HS}}=10 \mathrm{nT}\right)$, $\left(\theta=20^{\circ}, B_{\mathrm{HS}}=100 \mathrm{nT}\right),\left(\theta=45^{\circ}, B_{\mathrm{HS}}=100 \mathrm{nT}\right)$ (see Sect. 2). For cases where only a lower limit to $\nu_{\mathrm{c}}$ is available (Table 1), the value of $\eta$ is a lower limit, as indicated by the symbol $>$ near the top, and vice versa. Note that all values of $\eta>3$ are truncated at $\eta=3$ in the plots.

\section{Discussion}

In this study, our aim was to enquire if the reported observations of optical/near-IR synchrotron emission from the hotspots of double radio sources can be explained without having to invoke in situ acceleration of relativistic electrons inside the hotspots. In situ acceleration would be necessary if the parameter $\eta$ for a given hotspot were found to be well above unity for all combinations of the adopted plausible values of $\theta, B_{\mathrm{HS}}$ and $\Gamma_{\mathrm{j}}$. It is seen from Fig. 1 that for all the hotspots, at least one combination of the adopted plausible values of $\theta$ and $B_{\mathrm{HS}}$ yields $\eta \lesssim 1$, for values of $\Gamma_{\mathrm{j}}$ up to 5 . Even $\Gamma_{\mathrm{j}}=10$ is consistent with $\eta \lesssim 1$, with the possible exception of the hotspots in $3 \mathrm{C} 351$; however, in both these cases the estimate of $\nu_{\mathrm{c}}$ is very uncertain (Class III, Table 1). It is thus apparent that if the overall synchrotron losses of electrons during their transit through the jet remain small compared with the (inevitable) energy losses due to Compton upscatterings of the CMB photons, the particles accelerated within the central nucleus can account for the synchrotron optical/near-IR emission detected from the hotspots.

The negligible amount of synchrotron losses within the jet, assumed here, is in accord with some energy transport scenarios proposed earlier (e.g., Owen et al. 1989; Begelman et al. 1994; Kundt 1996; Heinz \& Begelman 1997). It is also consistent with the recent evidence in support of the large-scale jets comprising a fast relativistic spine surrounded by a more dissipative, slower moving layer of synchrotron plasma (e.g., Laing et al. 1999; Swain et al. 1998; Bridle 1996; also, Chiaberge et al. 2000b; Giovannini et al. 1999; Carilli et al. 1996; Laing 1993; Komissarov 1990), although it should be noted that most of the evidence for this spine/sheath structure has been adduced for the weaker FR I sources. Nonetheless, some FR II sources do appear to behave similarly (e.g. Chiaberge et al. 2000a; Ghisellini \& Celotti 2001). 
It may be emphasized that the main conclusion of this work does not demand assuming $B_{\mathrm{j}}=0$. From Table 1 , the equivalent magnetic field of the $\mathrm{CMB}$, as would be experienced by the relativistic jets of the sources in our sample, has a mean value of $2.8 \mathrm{nT}$. Thus, as long as the volume-averaged magnetic field is only up to $\sim 1 \mathrm{nT}$, the synchrotron losses in the jet will be minor in comparison to the inverse Compton losses against the CMB photons. Here it may be recalled that for the kpc-scale radio jets in moderately powerful radio galaxies, the typically adopted average magnetic field is $\sim 1 \mathrm{nT}$ (Bicknell 1995). Moreover, this value of $B_{\mathrm{j}}$ is an average over the jet's cross section. If the magnetic field is concentrated near the jet's outer layers due to surface interactions, its strength there can be considerably higher than $1 \mathrm{nT}$ without forcing the synchrotron losses to become the primary mechanism of energy loss in the overall jet flow. Further, our picture invoking the dominance of inverse Compton losses within the jet obviously does admit dissipation within the jet. This is evident, for instance, from the gradual spectral softening along the $3 \mathrm{C} 273$ jet, as inferred from a comparison of its radio, optical and X-ray images (Harris 2001).

By far the strongest case for in situ acceleration reported in the literature is that of the western lobe of the radio galaxy Pictor $A$ (Table 1 ). In this lobe, the linearly polarized optical synchrotron radiation is seen to extend up to $\sim 10 \mathrm{kpc}$ from the hotspot (Perley et al. 1997; also, Thomson et al. 1995; Röser \& Meisenheimer 1987) and broadly co-spatial X-ray emission (of poorly understood origin) has recently been detected by Wilson et al. (2001) using $C H A N D R A$. As noted by these authors, the level of $\mathrm{X}$-ray emission is much above a simple extrapolation of the radio-optical spectrum, which shows a clear break in the near-IR region. Hence the X-ray flux cannot be synchrotron radiation from the same electron population. At the same time, inverse-Compton upscattering of the CMB photons by this electron population is also an unlikely explanation for the X-ray emission, for the X-ray spectral slope is found to be significantly different from that of the radio spectrum of the hotspot (Wilson et al. 2001). Thus, the source of the X-ray emission is unclear at present. A similar situation may be present in the northern hotspot of 3C 390.3, whose $V, R$, and $I$ photometry indicates a spectral cut-off near $3 \times 10^{14} \mathrm{~Hz}$ (Prieto \& Kotilainen 1997). Hence any smooth extrapolation of this hotspots' spectrum would fall much below the level of X-ray detection using ROSAT (Harris et al. 1998), which is way above the level expected from Compton up-scatterings of either the internally generated photons or the CMB photons (Harris et al. 1998; Prieto \& Kotilainen 1997).

At the same time, the large spatial extent of the optical synchrotron emission (up to $\sim 10 \mathrm{kpc}$ for Pictor A, see above) observed from a few radio lobes seems highly intriguing, given that the estimated synchrotron lifetimes of the optically radiating electrons in the lobe are only of the order of $10^{3} \mathrm{yr}$ (Röser \& Meisenheimer 1987; Perley et al. 1997), during which the electrons cannot travel more than $\sim 1 \mathrm{kpc}$ from the hotspot. This apparent discrepancy may be resolved, if in reality, the magnetic field inside the lobes is concentrated in filaments, leaving large volumes with very weak field. As emphasized by Scheuer (1989), relativistic electrons could then travel much farther away through the essentially field-free regions of the lobe, without decaying appreciably in energy, and thus retaining enough energy to generate widespread optical synchrotron emission upon interacting with magnetized filaments (see also, Gopal-Krishna 1980; Siah \& Wiita 1990; Tribble 1993; Eilek et al. 1997). This picture of highly filamentary magnetic field within the lobe is, in fact, supported by the radio fine structure detected in the VLA images of several radio galaxies. For instance, in the case of Cygnus A, only 10\% of the lobe volume is estimated to be occupied by the magnetized filaments (Perley et al. 1984).

In summary, by adopting the generally accepted range of values for physical parameters of classical double radio sources (such as the hotspot's magnetic field, the jet's bulk speed and the radio axis orientation angle), we have argued that while in situ acceleration of relativistic electrons inside the hotspots/lobes of radio galaxies may seem an attractive possibility, the observations reported thus far do not make it a compelling requirement.

Acknowledgements. It is a pleasure to thank R. Nityananda for fruitful discussions and the anonymous referee for helpful suggestions. This work was supported in part by Research Program Enhancement funds at Georgia State University and by the Council on Science and Technology at Princeton.

\section{Appendix A:}

A quantity of central importance in this paper is the timescale $\tau$ for the ultrarelativistic electrons in the jet to cool via the emission of inverse-Compton radiation. In the frame of the central object, which is the "laboratory" frame, the jet has speed $c \beta_{\mathrm{j}}$ and bulk Lorentz factor $\Gamma_{\mathrm{j}}=\left(1-\beta_{\mathrm{j}}^{2}\right)^{-1 / 2}$, and the CMBR is isotropic. We wish to calculate $\tau$ in this frame. The total inverseCompton power emitted per unit volume by an electron with Lorentz factor $\gamma$ and speed $c \beta=c \sqrt{1-\gamma^{-2}}$ as measured in the lab frame is (e.g., Rybicki \& Lightman 1979)

$P_{\text {compt }}(\gamma)=\frac{4}{3} \sigma_{\mathrm{T}} c \gamma^{2} \beta^{2} u_{\mathrm{rad}}$,

where $u_{\text {rad }}$ is the lab-frame energy density of the background radiation and $\sigma_{\mathrm{T}}$ is the Thomson cross section. To determine the total inverse-Compton power per unit volume in the lab frame, we must integrate Eq. (A1) over the electron distribution as seen in that frame. For simplicity, we assume that the electron distribution is isotropic and monoenergetic as viewed in the frame of the jet. Naturally, in the lab frame the electron distribution is neither monoenergetic nor isotropic due to the Lorentz boost. Hence we must perform a Lorentz transformation of the electron distribution in order to integrate Eq. (A1). It is convenient for this purpose to introduce the "electron intensity" $I_{\epsilon}$, which is defined so that in time $\mathrm{d} t$, the energy passing 
through a differential cylinder with face area $\mathrm{d} A$ due to electrons with energy in the range $\mathrm{d} \epsilon$ propagating in the differential solid angle $\mathrm{d} \Omega$ is given by $\mathrm{d} E=I_{\epsilon} \mathrm{d} \Omega \mathrm{d} A \mathrm{~d} t \mathrm{~d} \epsilon$. The total electron number density $n$ and energy density $u$ in the lab frame are expressed in terms of $I_{\epsilon}$ by the integrals

$n=\iint_{0}^{\infty} \frac{I_{\epsilon}}{c \epsilon} \mathrm{d} \epsilon \mathrm{d} \Omega, \quad u=\iint_{0}^{\infty} \frac{I_{\epsilon}}{c} \mathrm{~d} \epsilon \mathrm{d} \Omega$,

respectively.

The electron Lorentz factor in the jet frame $\left(\gtrsim 10^{4}\right)$ greatly exceeds the jet bulk Lorentz factor $\Gamma_{\mathrm{j}} \lesssim 10$, and therefore the electrons are ultrarelativistic in both the jet and lab frames. It follows that the electron intensity transforms in exactly the same manner as the usual radiation intensity. By analogy with the radiation case, we can consequently relate the intensities in the two frames using

$\frac{I_{\epsilon}(\epsilon)}{\epsilon^{3}}=\frac{I_{\epsilon}^{\prime}\left(\epsilon^{\prime}\right)}{\epsilon^{\prime 3}}$,

where $\epsilon$ is the electron energy and primed quantities are measured in the jet frame. The electron energies are likewise related by the formula

$\epsilon^{\prime}=\epsilon \Gamma_{\mathrm{j}}\left(1-\beta_{\mathrm{j}} \cos \phi\right)$,

where $\phi$ is the angle of propagation of the electron relative to the axis of the jet, as measured in the lab frame.

Since the electron distribution is isotropic and monoenergetic in the jet frame, the electron intensity in that frame is given simply by

$I_{\epsilon}^{\prime}\left(\epsilon^{\prime}\right)=\frac{c \epsilon^{\prime} n^{\prime} \delta\left(\epsilon^{\prime}-\epsilon_{*}\right)}{4 \pi}$,

where $\epsilon_{*}$ is the energy of each electron in the jet frame and $n^{\prime}$ is the jet-frame electron number density. Utilizing Eqs. (A3)-(A5), we can now express the lab-frame electron intensity as

$I_{\epsilon}(\epsilon)=\frac{n^{\prime} c \epsilon}{4 \pi \Gamma_{\mathrm{j}}^{2}\left(1-\beta_{\mathrm{j}} \cos \phi\right)^{2}} \delta\left[\epsilon \Gamma_{\mathrm{j}}\left(1-\beta_{\mathrm{j}} \cos \phi\right)-\epsilon_{*}\right]$.

Before utilizing this result to calculate the lab-frame number and energy densities, it is convenient to transform the $\delta$-function using

$$
\begin{aligned}
& \delta\left[\epsilon \Gamma_{\mathrm{j}}\left(1-\beta_{\mathrm{j}} \cos \phi\right)-\epsilon_{*}\right] \\
& \quad=\delta\left[\epsilon-\frac{\epsilon_{*}}{\Gamma_{\mathrm{j}}\left(1-\beta_{\mathrm{j}} \cos \phi\right)}\right] \frac{1}{\Gamma_{\mathrm{j}}\left(1-\beta_{\mathrm{j}} \cos \phi\right)} .
\end{aligned}
$$

Substituting this expression into Eqs. (A2) and integrating over $\epsilon$ yields

$n=\int \frac{n^{\prime}}{4 \pi \Gamma_{\mathrm{j}}^{3}\left(1-\beta_{\mathrm{j}} \cos \phi\right)^{3}} \mathrm{~d} \Omega$,

$u=\int \frac{n^{\prime} \epsilon_{*}}{4 \pi \Gamma_{\mathrm{j}}^{4}\left(1-\beta_{\mathrm{j}} \cos \phi\right)^{4}} \mathrm{~d} \Omega$.
Setting $d \Omega=2 \pi \sin \phi d \phi$ and integrating over $\phi$ in the range $0 \leq \phi \leq \pi$, we obtain for the lab-frame number and energy densities the exact results

$$
n=n^{\prime} \Gamma_{\mathrm{j}}, \quad u=u^{\prime} \Gamma_{\mathrm{j}}^{2}\left(1+\frac{1}{3} \beta_{\mathrm{j}}^{2}\right),
$$

where $u^{\prime}=n^{\prime} \epsilon_{*}$ is the jet-frame electron energy density.

Next we calculate the total power per unit volume emitted by the electrons due to inverse-Compton scattering of the background radiation, given by

$\dot{u}=\iint_{0}^{\infty} P_{\text {compt }}(\gamma) \frac{I_{\epsilon}}{c \epsilon} \mathrm{d} \epsilon \mathrm{d} \Omega$,

where $\gamma=\epsilon /\left(m_{\mathrm{e}} c^{2}\right)$ and $m_{\mathrm{e}}$ is the electron rest mass. Substitution using Eqs. (A1), (A6), and (A7) yields, upon integration

$\dot{u}=\frac{4}{3} \frac{c \sigma_{\mathrm{T}} u_{\mathrm{rad}}}{m_{\mathrm{e}}^{2} c^{4}} u^{\prime} \epsilon_{*} \Gamma_{\mathrm{j}}^{3}\left(1+\beta_{\mathrm{j}}^{2}\right)$.

Note that in deriving this expression we have set $\beta=1$ in Eq. (A1), which is an extremely accurate approximation for the ultrarelativistic electrons of interest here. Our final step is to compute the inverse-Compton loss timescale, $\tau \equiv u / \dot{u}$, given by

$\tau=\frac{3 m_{\mathrm{e}} c^{2}}{4 c \sigma_{\mathrm{T}} u_{\mathrm{rad}}} \frac{1}{\gamma_{*} \Gamma_{\mathrm{j}}}\left[\frac{1+\beta_{\mathrm{j}}^{2} / 3}{1+\beta_{\mathrm{j}}^{2}}\right]$,

where $\gamma_{*} \equiv \epsilon_{*} /\left(m_{\mathrm{e}} c^{2}\right)$ is the Lorentz factor of the monoenergetic electrons in the jet frame (denoted $\gamma$ in the main text). Equation (A11) gives the value of $\tau$ measured in the lab frame by virtue of the definitions of $u$ and $\dot{u}$, and the factor in brackets provides the correction factor quoted following Eq. (6).

\section{References}

Arshakian, T. G., \& Longair, M. S. 2000, MNRAS, 311, 846

Barthel, P. D. 1989, ApJ, 336, 606

Begelman, M. C., Blandford, R. D., \& Rees, M. J. 1984, Rev. Mod. Phys., 56, 255

Begelman, M. C., Rees, M. J., \& Sikora, M. 1994, ApJ, 429, L57

Bell, A. R. 1978, MNRAS, 182, 147

Bicknell, G. V. 1995, ApJS, 101, 29

Biermann, P. L., \& Strittmatter, P. A. 1987, ApJ, 322, 643

Biretta, J. A., Sparks, W. B., \& Macchetto, F. 1999, ApJ, 520, 621

Blandford, R. D., \& Rees, M. J. 1974, MNRAS, 169, 395

Bridle, A. H. 1996, in Energy Transport in Extragalactic Radio Sources, ed. P. E. Hardee, A. H. Bridle, \& J. A. Zensus (ASP, San Francisco), 383

Bridle, A. H., Hough, D. H., Lonsdale, C. J., Burns, J. O., \& Laing, R. A. 1994, AJ, 108, 766

Carilli, C. L., Perley, R. A., Bartel, N., \& Sorathia, B. 1996, in Energy Transport in Extragalactic Radio Sources, ed. P. E. Hardee, A. H. Bridle, \& J. A. Zensus (ASP, San Francisco), 287

Chiaberge, M., Capetti, A., \& Celotti, A. 2000a, A\&A, 355, 873 
Chiaberge, M., Celotti, A., Capetti, A., \& Ghisellini, G. 2000b, A\&A, 358, 104

Contopoulos, J., \& Kazanas, D. 1995, ApJ, 441, 521

Crane, P., Tyson, J. A., \& Saslaw, W. C. 1983, ApJ, 265, 681

Dar, A., \& Laor, A. 1987, ApJ, 478, L5

Dermer, C. D., \& Schlickeiser, R. 1993, ApJ, 416, 458

Dermer, C. D., \& Schlickeiser, R. 1994, ApJS, 90, 945

Dreher, J. W., Simkin, S. M. 1986, AJ, 91, 58

Drury, L.O'C. 1983, Rep. Prog. Phys., 46, 973

Eichler, D., \& Wiita, P. J. 1978, Nature, 274, 38

Eilek, J. A., Melrose, D. B., \& Walker, M. A. 1997, ApJ, 483, 282

Fanaroff, B. L., \& Riley, J. M. 1974, MNRAS, 167, 31P

Felton, J. E. 1968, ApJ, 151, 861

Garrington, S. T., Leahy, J. P., Conway, R. G., Laing, R. A., 1988, Nature, 331, 147

Ghisellini, G., \& Celloti, A. 2001, in Issues of Unifications of AGNs, ed. R. Maiolino, A. Marconi, \& N. Nagar, in press [astro-ph/0108110]

Giovannini, G., Taylor, G. B., Arbizzani, E., et al. 1999, ApJ, 522,101

Gopal-Krishna 1980, A\&A, 81, 328

Gopal-Krishna, Kulkarni, V. K., \& Wiita, P. J. 1996, ApJ, 463, L1

Hardcastle, M. J., Birkinshaw, M., \& Worrall, D. M. 2001, MNRAS, in press [astro-ph/0101240]

Hargrave, R. J., \& Ryle, M. 1976, MNRAS, 175, 481

Harris, D. E. 2001, in Particles and Fields in Radio Galaxies, ed. R. A. Laing, \& K. A. Blundell (ASP, San Francisco), in press

Harris, D. E., Carilli, C. L., \& Perley, R. A. 1994, Nature, 367, 713

Harris, D. E., Leighly, K. M., \& Leahy, J. P. 1998, ApJ, 499, L149

Harris, D. E., Nulsen, P. E. J., Ponman, T. J., et al. 2000, ApJ, 530, L81

Heavens, A. F., \& Meisenheimer, K. 1987, MNRAS, 225, 335

Heinz, S., \& Begelman, M. C. 1997, ApJ, 490, 653

Hiltner, P. R., Meisenheimer, K., Röser, H.-J., Laing, R. A., \& Perley, R. A. 1994, A\&A, 286, 25

Jenkins, C. J., Pooley, G. G., \& Riley, J. M. 1977, Mem. RAS, 84,61

Keel, W. C. 1988, ApJ, 329, 532

Keel, W. C., \& Martini, P. 1995, AJ, 109, 2305

Kirk, J. G., \& Schneider, P. 1987, ApJ, 322, 256

Komissarov, S. S. 1990, Sov. Astr. Lett., 16, 284

Kronberg, P. 1976, ApJ, 203, L47

Kronberg, P., van den Bergh, S., \& Button, S. 1977, AJ, 82, 1039

Kundt, W. 1986, in Astrophysical Jets and Their Engines, ed. W. Kundt (Kluwer, Dordrecht), 1

Kundt, W. 1996, in Jets from Stars and Galactic Nuclei, ed. W. Kundt (Springer, Berlin), 1

Lähteenmäki, A., \& Valtaoja, E. 1999, AJ, 117, 1168

Laing, R. A. 1988, Nature, 331, 149

Laing, R. A. 1993, in STScI Symp. 6, Astrophysical Jets, ed. D. Burgarella, M. Livio, \& C. O'Dea (Cambridge University Press), 95

Laing, R. A., Parma, P., de Ruiter, H. R., \& Fanti, R. 1999, MNRAS, 306, 513

Lang, K. R. 1999, Astrophysical Formulae (Springer, New York)

Leahy, J. P., \& Perley, R. A. 1995, MNRAS, 277, 1097
Lelievre, G., \& Wlerick, G. 1975, A\&A, 42, 293

Linfield, R., \& Perley, R. 1984, ApJ, 279, 60

Longair, M. S. 1981, High Energy Astrophysics (Cambridge University Press), 279

Looney, L. W., \& Hardcastle, M. J. 2000, ApJ, 534, 172

Mannheim, K. 1993, A\&A, 269, 67

Mannheim, K., \& Biermann, P. L., \& Krülls, W. M. 1991, A\&A, 251, 723

Mastichiadis, A., \& Kirk, J. G. 1995, A\&A, 295, 613

Meisenheimer, K. 1996, in Jets from Stars and Galactic Nuclei, ed. W. Kundt (Springer, Berlin), 57

Meisenheimer, K., \& Röser, H.-J. 1986, Nature, 319, 459

Meisenheimer, K., Röser, H.-J., Hiltner, P. R., et al. 1989, A\&A, 219, 63

Meisenheimer, K., Yates, M. G., \& Röser, H.-J. 1997, A\&A, 325,57

Okayasu, R., Ishiguro, M., \& Tabara, H. 1992, PASJ, 44, 335

Orr, M. J. L., \& Browne, I. W. A. 1982, MNRAS, 200, 1067

Owen, F. N., Hardee, P. E., \& Cornwell, T. J. 1989, ApJ, 340, 698

Perley, R. A., Dreher, D. W., \& Cowan, J. J. 1984, ApJ, 285, L35

Perley, R. A., Röser, H.-J., \& Meisenheimer, K. 1997, A\&A, 328,12

Polatidis, A. G., Wilkinson, P. N., Xu, W., et al. 1995, ApJS, 98,1

Prieto, M. A., \& Kotilainen, J. K. 1997, ApJ, 491, L77

Rees, M. J. 1967, MNRAS, 135, 345

Rees, M. J. 1971, Nature, 229, 312

Ridgway, S. E., \& Stockton, A. 1997, AJ, 114, 511

Röser, H.-J., \& Meisenheimer, K. 1987, ApJ, 314, 70

Röser, H.-J., \& Meisenheimer, K. 1991, A\&A, 252, 458

Röser, H.-J., Meisenheimer, K., Neumann, M., Conway, R. G., \& Perley, R. A. 2000, A\&A, 360, 99

Rybicki, G. B., \& Lightman, A. P. 1979, Radiative Processes in Astrophysics (Wiley, New York)

Saikia, D. J., Salter, C. J., \& Muxlow, T. W. B. 1987, MNRAS, 224,911

Saslaw, W. C., Tyson, A., \& Crane, P. 1978, ApJ, 222, 435

Scheuer, P. A. G. 1987, in Astrophysical Jets and their Engines, ed. W. Kundt (Reidel, Dordrecht), 129

Scheuer, P. A. G. 1989, in Hot Spots in Extragalactic Radio Sources, ed. K. Meisenheimer, \& H.-J. Röser (Springer, Berlin), 159

Scheuer, P. A. G. 1995, MNRAS, 277, 331

Scott, D., Silk, J., Kolb, E. W., \& Turner, M. S. 2000, in Allen's Astrophysical Quantities, ed. A. N. Cox (Springer, New York), 664

Shklovskii, I. S. 1984, Sov. Astr., 28, 489

Siah, M. J., \& Wiita, P. J. 1990, ApJ, 363, 411

Simkin, S. 1978, ApJ, 222, L55

Simkin, S. 1979, ApJ, 234, 56

Subramanian, P., Becker, P. A., \& Kazanas, D. 1999, ApJ, 523, 203

Swain, M. R., Bridle, A. H., \& Baum, S. A. 1998, ApJ, 507, L29

Thomson, R. C., Crane, P., \& Mackay, C. D. 1995, ApJ, 446, L93

Tribble, P. C. 1993, MNRAS, 261, 57

Valtonen, M. J., \& Heinämäki, P. 2000, ApJ, 530, 107

Vermeulen, R. C., \& Cohen, M. H. 1994, ApJ, 430, 467

Wardle, J. F. C., \& Aaron, S. E. 1997, MNRAS, 286, 425

Wilson, A. S., Young, A. J., \& Shopbell, P. L. 2000, ApJ, 544, L27

Wilson, A. S., Young, A. J., \& Shopbell, P. L. 2001, ApJ, 547, 740 